\title{
Digitalisierung in der Schule - Datenschutz mitdenken
}

\author{
Marit Hansen
}

\begin{abstract}
An Schulen werden hohe Erwartungen gestellt, auch im Bereich der Digitalisierung. Schon von Kindesbeinen an sollen die Kinder und Jugendlichen mit Technik und digitalen Informationsangeboten umgehen lernen. Jedoch stehen noch nicht überall datenschutzkonforme Lösungen zur Verfügung, sodass Schülerinnen und Schüler ebenso wie Lehrkräfte Risiken in Bezug auf ihre personenbezogenen Daten und Privatsphäre ausgesetzt werden. Hier besteht Nachholbedarf. Wichtig dafür ist es, Datenschutz mitzudenken und in neue Anwendungen von Anfang an einzubauen. Dies betrifft sowohl die Basis-Arbeitsmittel für Unterricht und Schulverwaltung als auch pädagogische Lehrmaterialien. Hilfestellungen und Austausch untereinander können dazu führen, dass nicht jede Schule selbst das Rad neu erfinden muss, sondern dass Best Practices standardisiert zur Verfügung gestellt werden.
\end{abstract}

\section{Startpunkt der Schulen ${ }^{1}$}

Schule und Digitalisierung. Und Datenschutz. Passt dies alles zusammen? Noch nicht so wirklich: Der Schulalltag sieht anders aus. Während für Schülerinnen und Schüler zumindest auf den weiterführenden Schulen der Einsatz von Smartphones, das Abrufen von Fotos, Videos und Musik aus dem Internet sowie die Benutzung von Sozialen Medien in ihrem Privatleben eine Selbstverständlichkeit ist, läuft der Unterricht zumeist weit weniger digital(isiert) ab. Dabei wird von den Schulen erwartet, dass sie die Kinder und Jugendlichen auf das Leben vorbereiten, auch auf das Leben in der zunehmend digitalisierten Informationsgesellschaft. Dass sie Medienkompetenz - sowohl für das Nutzen von Angeboten als auch für das Bereitstellen von Inhalten - lehren. Dass Informatik-Sachverhalte und

1 Anmerkung: Dieser Text entstand auf Basis des Workshops „Digitalisierung in der Schule - Datenschutz mitdenken“ auf der Jahrestagung 2019 des Forum Privatheit. 
technisch geprägte Themen vermittelt werden, und zwar an alle (Lautebach 2018).

Die besondere Wichtigkeit von Digitalisierung in Schulen zeigt sich in Situationen, in denen der Schulunterricht nicht physisch stattfinden kann. So zeigten sich im März 2020, als die Schulen in Deutschland aufgrund der Corona-Pandemie weitgehend geschlossen wurden und auf Fernunterricht ausweichen mussten, erhebliche Probleme im Homeschooling. Laut der internationalen Vergleichsstudie „International Computer and Information Literacy Study" (ICILS) hat Deutschland Nachholbedarf, beispielsweise beim schulischen WLAN für die Internet-Nutzung, wie dies in Deutschland 2018 erst $25 \%$ der Schulen ermöglichten, während dies in Dänemark an nahezu jeder Schule Standard ist (Fraillon et al. 2020). In einer repräsentativen telefonischen Befragung von 1.003 Personen in Deutschland ab 16 Jahren, darunter 269 Eltern schulpflichtiger Kinder, im August 2020 hat der deutsche Digitalverband „Bitkom“ ermitteln lassen, wie die Bevölkerung und insbesondere Eltern den Stand der digitalen Bildung einschätzt (Bitkom Research 2020). Fast alle Eltern wollten digitalen Unterricht, ist eines der Ergebnisse. Demnach stünde die Notwendigkeit der Ausstattung mit digitalen Technologien wie Computer, Smartboards oder Tablets im Vordergrund (Zustimmung von $93 \%$ der befragten Eltern; die Werte für die Gesamtbevölkerung liegen jeweils einige Prozentpunkte darunter); dadurch sollten die Schüler auf das Leben und Arbeiten in der digitalen Welt vorbereitet werden (Zustimmung von $79 \%$ der Eltern). Dass digitale Technologien es den Lehrkräften ermöglichten, individueller auf einzelne Schülerinnen und Schüler einzugehen, wurde in der Umfrage nicht im selben Maße bestätigt (Zustimmung von $43 \%$ der Eltern (Bitkom Research 2020: 7)). Maßnahmen, um die Digitalisierung der Schulen voranzubringen, wurden sowohl im Bereich der Verbesserung der technischen Ausstattung der Schulen (Zustimmung von 96\% der Eltern (Bitkom Research 2020: 9)) als auch im Bereich der Lehrerfortbildungen und Anpassung der Lehrpläne an die Möglichkeiten der Digitalisierung (Zustimmung jeweils $93 \%$ der Eltern (Bitkom Research 2020: 10)) gesehen.

Markig heißt es vom Bitkom-Präsidenten Achim Berg, dass die „Corona-Krise [...] unser Bildungssystem vor eine Zerreißprobe gestellt" hat. „Die massiven Verunsicherungen durch Behörden und Datenschutzbeauftragte haben dann auch noch die digitalen Vorreiter unter den Schulen und Lehrkräften ausgebremst.“ (Bitkom 2020)

Zwar enthält der online verfügbare Foliensatz zur Umfrage von Bitkom Research gar keine Aussagen zu Datenschutz, auch nicht zu weiteren öfter genannten Problemen wie beispielsweise zur notwendigen Priorisierung in den Schulen vor Ort, wenn bauliche oder Hygienemängel behoben wer- 
den müssen. Aber die Verunsicherung scheint groß zu sein. Nicht erst seit der Corona-Pandemie: Schon im April 2018 äußerte sich der Lehrer Matthias Förtsch in einer Kolumne zu einer angeblichen „Diktatur des Datenschutzes“: „Was ist wichtiger? Die zeitgemäße Bildung aller Schülerinnen und Schüler oder das möglichst geringe Risiko eines potenziellen Datenmissbrauchs? Beides zusammen geht (bislang) nicht: Die Europäische Datenschutz-Grundverordnung (DSGVO) gewährleistet Datensicherheit, Datenschutzvorschriften insgesamt können aber einem modernen, digitalen und vernetzten Schulalltag auch im Weg stehen“ (Förtsch 2020).

Hier mögen Aspekte von Datensicherheit und Datenschutz etwas durcheinander geraten zu sein, denn die Datenschutz-Grundverordnung enthält Regeln zum Schutz personenbezogener Daten, wobei die Sicherheit - also Vertraulichkeit, Integrität und Vertraulichkeit - nur eine der zahlreichen Anforderungen ist, die bei einer fairen Verarbeitung zu berücksichtigen sind. Schön wäre natürlich, wenn bereits - wie bei ihrer Einführung versprochen - die DSGVO tatsächlich ihre Wirkung dahingehend entfaltet hätte, dass sämtlich Angebote und Dienstleistungen im europäischen Markt, die mit personenbezogenen Daten zu tun haben, rechtskonform gestaltet wären und damit einem Datenmissbrauch der Riegel vorgeschoben wäre. Wäre dies so, würde es die Auswahl von Systemen für Schulverwaltung oder Homeschooling und ihren rechtskonformen Einsatz stark vereinfachen. Die Realität sieht anders aus. Dass aber dennoch Datenschutz und Schulalltag keine Gegensätze sein müssen, wird im Folgenden beleuchtet.

Abschnitt 2 gibt einen Überblick über generelle Datenschutzanforderungen. Dass für „Schule“ kein „One-size-fits-all“-Ansatz funktioniert, rückt Abschnitt 3 ins Bewusstsein, der verschiedene Charakteristika je nach Einsatzszenario und die Abgrenzungsnotwendigkeiten der Akteure je nach ihrer individuellen Rolle beleuchtet. Wesentliche Herausforderungen und Trends für Digitalisierung aus Datenschutzsicht werden in Abschnitt 4 dargestellt. Schließlich gibt Abschnitt 5 Empfehlungen für Digitalisierung in der Schule, bei dem Datenschutz von Anfang an mitgedacht wird.

\section{Datenschutzanforderungen - ganz kurz}

Beim Datenschutz geht es um den Schutz der Menschen und ihrer Persönlichkeitsrechte. In der Datenschutz-Grundverordnung steht in Artikel 1 in den Absätzen 1 und 2: 
„(1) Diese Verordnung enthält Vorschriften zum Schutz natürlicher Personen bei der Verarbeitung personenbezogener Daten und zum freien Verkehr solcher Daten.

(2) Diese Verordnung schützt die Grundrechte und Grundfreiheiten natürlicher Personen und insbesondere deren Recht auf Schutz personenbezogener Daten.“

Mit der Digitalisierung werden immer mehr Daten verarbeitet, darunter viele mit Personenbezug. Deswegen spielen Datenschutzanforderungen häufig eine Rolle, wenn Prozesse digitalisiert werden oder wenn die Akteure sich digitaler Dienstleistungen bedienen, z. B. über Angebote im Internet. $\mathrm{Zu}$ den Datenschutzrisiken gehören nicht nur unberechtigte $\mathrm{Zu}$ griffe auf die Daten, sondern auch unfaire Arten der Datensammlung oder -auswertung, z. B. bei Überwachung oder Beobachtung des Verhaltens der Menschen. Anhand von personenbezogenen Daten lassen sich viele Schlüsse über die Personen ziehen, auf Basis derer Entscheidungen getroffen werden können. Dies ist nicht in jedem Fall illegal oder illegitim, aber stets ist Aufmerksamkeit geboten, um Fairness der Verarbeitung zu gewährleisten und beispielsweise Manipulation auszuschließen.

Schulen müssen selbstverständlich Datenschutzanforderungen in ihren eigenen papierenen oder digitalen Verarbeitungen einhalten. Zusätzlich gehört Datenschutz in den Unterricht, um einerseits die Grundsätze, die sich aus den Grundrechten und Menschenrechten ableiten, und andererseits heutige oder kommende Datenverarbeitungen mit ihren Risiken und Gestaltungsmöglichkeiten verständlich zu machen.

Kernstück der DSGVO ist Artikel 5 mit den Grundsätzen für die Verarbeitung personenbezogener Daten. In Absatz 1 werden die folgenden Grundsätze aufgelistet:
a) Rechtmäßigkeit, Verarbeitung nach Treu und Glauben, Transparenz
b) Zweckbindung
c) Datenminimierung
d) Richtigkeit
e) Speicherbegrenzung
f) Integrität und Vertraulichkeit

„Rechtmäßigkeit“ bedeutet: Für die Verarbeitung personenbezogener Daten benötigt man eine Rechtsgrundlage, beispielsweise ein Gesetz, eine Einwilligung oder einen Vertrag.

Die Verarbeitung muss fair erfolgen („Treu und Glauben“). Wichtig ist auch die Transparenz der Verarbeitung, also eine verständliche Information für die betroffenen Personen. Vor der Erhebung von Daten muss man 
sich bewusst machen, welchen Zwecken die Verarbeitung dienen soll, denn davon hängt $a b$, für welche Zwecke die personenbezogenen Daten verwendet werden dürfen („Zweckbindung“), welche Daten für die Zwecke erforderlich sind, sodass keine weiteren Daten verarbeitet werden („Datenminimierung“), für wie lange die Daten in identifizierender Form benötigt werden, sodass sie danach zu löschen sind („Speicherbegrenzung“). Außerdem muss der Verantwortliche Sorge für die „Richtigkeit“ der Daten und für die Datensicherheit (insbesondere „Integrität und Vertraulichkeit“) tragen, damit sie nicht unbefugt zugänglich werden.

Jede Verarbeitung personenbezogener Daten bedeutet ein Risiko; deswegen muss der Verantwortliche - das ist derjenige, der über die Zwecke und Mittel der Verarbeitung von personenbezogenen Daten entscheidet sich dessen bewusst sein und geeignete Maßnahmen treffen, um das Risiko ausreichend einzudämmen.

Da der Schulbereich in Deutschland föderal geregelt ist, gilt für die Verarbeitung von Schülerdaten das Schulgesetz des jeweiligen Bundeslandes, ergänzend das Landesdatenschutzgesetz und stets auch die DSGVO. In Schleswig-Holstein wird das Schulgesetz von einer Schul-Datenschutzverordnung flankiert, die ebenfalls Regeln zum Umgang mit personenbezogenen Daten enthält und damit konkretisierende Anforderungen und Hilfestellungen enthält. Schulverwaltungen und Lehrkräfte sind zumeist gewohnt, mit ihren Schulgesetzen zu arbeiten, da sich auf dieser Basis vielerlei Prozesse - beispielsweise das Führen von Verwaltungsakten in der Schule - etabliert haben. Es empfiehlt sich, auch bei der zunehmenden Digitalisierung zu standardisierten Lösungen zu kommen, damit nicht jede Lehrkraft oder jede Schule das Rad von Neuem erfinden muss.

\section{Abgrenzungsnotwendigkeiten in der Schule}

Schule ist nicht Privatvergnügen, Schule ist aber auch nicht typische Behörde. An Schule und Lehrkräfte stellen sich hohe Erwartungen. Umso wichtiger ist es, sich bewusst zu machen, welche Regeln gerade gelten, welche Rolle einzuhalten ist und wo Grenzziehungen nötig sind.

\subsection{Schule: Verwaltung oder pädagogischer Auftrag}

Einerseits weist die Schule einen Verwaltungscharakter auf: Die Dokumentation über die Schülerin oder den Schüler muss korrekt geführt werden 
und nachvollziehbar sein, beispielsweise zu Leistungsnachweisen oder Abwesenheiten, die beispielsweise zeugnisrelevant sind. Dasselbe gilt für die Aktenführung in besonderen Situationen, die einen Schulverweis nach sich ziehen können und daher einer gerichtlichen Überprüfung standhalten können sollen.

Andererseits besteht ein pädagogischer Auftrag der Schulen, der nicht per bürokratischem Absolvieren der Unterrichtseinheiten gut zu erfüllen ist. Hier ist mehr individuelles Eingehen der Lehrkraft mit ihrer Persönlichkeit auf die Schülerinnen und Schüler vonnöten. Kreativität ist gefragt, Lehrinhalte können und sollen gerne mit aktuellen Geschehnissen in Verbindung gebracht werden.

\subsection{Schulische und außerschulische Veranstaltungen: Schutzraum oder reale Welt}

Generell sind die Sorgeberechtigten für ihre Kinder verantwortlich. In der Schule halten sich die Kinder aber ohne die Sorgeberechtigten auf. Das bedeutet zum einen, dass sie in der Schule vor schädigenden Einflüssen zu schützen sind, beispielsweise gemäß den Vorgaben des Jugendschutzes. Eine Lehrkraft darf daher im Unterricht keinen Film mit ungeeigneter Altersfreigabe zeigen.

Zum anderen sollen die Kinder ihre Persönlichkeit und die Befähigung zum Umgang mit der echten Welt auch im Schulbereich entwickeln können. Dies wäre aber kaum möglich, wenn die Schule ihren Schutzauftrag als vollständige Abschottung verstünde.

Während für schulische Veranstaltungen in der Regel eine Teilnahmepflicht der schulpflichtigen Kinder und Jugendlichen besteht, ist dies anders bei außerschulischen Veranstaltungen. Solche außerschulischen Aktivitäten können von der Schulleitung, Lehrkräften oder Klassengemeinschaften initiiert sein; manche ergeben sich auch beispielsweise in Zusammenarbeit mit Veranstaltern von Wettbewerben oder auf Anregung von Vereinen, Krankenkassen, Banken und Sparkassen sowie gemeinnützigen Einrichtungen. Selbstverständlich betrifft dies auch den Bereich der Digitalisierung, z. B. wenn Hardware oder Software gesponsort wird oder wenn besondere Digitalevents wie Wettbewerbe mit Bezug zur OnlineWelt anstehen, bei denen die Schülerinnen und Schüler mit ihren Namen und ggf. weiteren Daten angemeldet werden. Da je nach Bundesland unterschiedliche Regularien gelten, muss man sich über den schulischen oder außerschulischen Charakter im Klaren sein. Dies betrifft auch die Frage, ob eine Einwilligung der Eltern für die Teilnahme und die oft damit ver- 
bundene Weitergabe personenbezogener Daten notwendig ist und wem gegenüber sie abgegeben werden muss. Es ist ein Unterschied, ob es dabei nur um den Zweck des Teilnehmens geht oder ob zusätzlich Fotos oder Videos für den individuellen Gebrauch, für die Schul-Webseite oder für öffentliche Darstellungen in sozialen Medien gefertigt werden.

In den Schulgesetzen finden sich häufig Regelungen zum Umfang erlaubter Werbung - denn vollständig ausgeschlossen ist Werbung in den Klassenzimmern oder bei von der Schule initiierten oder unterstützten Veranstaltungen zumeist nicht. Es haben sich sogar Werbetreibende auf das Schulmarketing spezialisiert, denn schließlich handelt es sich um eine „konsumfreudige junge Zielgruppe“, die „über viele Milliarden an Kaufkraft" ${ }^{\text {" }}$ verfügt. Die damit verbunden Gefahren sehen auch Verbraucherschützer, die ein vollständiges Werbeverbot fordern (Verbraucherzentrale Bundesverband 2020). Oft übersehen werden die typischen Werbeeinblendungen beim Zeigen von Filmen aus Videoportalen oder beim Surfen im Internet, die möglicherweise sogar an die Zielgruppe oder an den jeweils verwendeten Nutzeraccount der Lehrkraft oder der einzelnen Schülerinnen und Schüler angepasst werden. Aus Datenschutzsicht sind hier die vorhandenen Datenspuren und ausgewerteten Nutzerprofile bedenkenswert; zusätzlich können Jugendschutzfragen relevant werden.

\subsection{Lehrkraft: Berufoder Privatmensch}

Engagierte Mitarbeitende bringen stets ein Stück ihrer Persönlichkeit mit ein und verstehen ihren Job nicht als ungeliebte Arbeit im vorgegebenen Zeitkorsett. Dies gilt auch für Lehrkräfte. Allerdings dürfen die Grenzen nicht verschwimmen, beispielsweise wenn Jugendliche für eine Lehrkraft schwärmen und auch privat den Kontakt zu ihrem Schwarm suchen. Hier empfiehlt sich das Einhalten der nötigen Distanz und eine unmissverständliche Ausdrucksweise. Das gilt auch für die digitale Welt mit zahlreichen privaten und öffentlichen Kommunikationswegen. Statt dass Lehrkräfte über private Nutzerkonten per E-Mail, Messenger oder in anderen sozialen Medien kommunizieren, bieten sich standardisierte Schul-E-Mail-Adressen oder andere Schul-Nutzerkonten an, bei denen stets der Kontext „Schule“ deutlich wird. Dies ist auch im Sinne der Fairness für das Privatleben der Lehrkräfte, denen damit eine Trennung zwischen Beruf und Freizeit ermöglicht wird und die beispielsweise generell „Freundschaftsanfragen“ in

2 https://www.grundschulmarketing.de/ (Abfrage am: 21.09.2020). 
sozialen Medien, in denen sie als Privatperson vertreten sind, von Schülerinnen und Schülern ablehnen sollten. Auch sollten Lehrkräfte überlegen, wann Gruppen-Nachrichten z. B. an die ganze Klasse sinnvoll sind und wann auch einmal eine individuelle (schulische) Kommunikation mit einzelnen Schülerinnen oder Schülern zu bevorzugen ist.

\section{Digitalisierungsschub: brave new school world?}

Wie die Bitkom-Umfrage herausarbeitet, ist der Zustand der Digitalisierung im Schulbereich noch verbesserungsbedürftig (Bitkom 2020). Mit der Corona-Pandemiesituation hat sich gezeigt, dass ein schnelles Umstellen des Unterrichts auf Online-Formate und eine Lehrer-Schüler-Kommunikation per elektronischen Medien nicht reibungslos funktioniert hat. Weder die Schulen noch die Haushalte der Schülerinnen und Schüler waren darauf vorbereitet. Es war Zufall, ob bei den Lehrkräften und Schülerinnen und Schülern geeignete Hard- und Software sowie eine ausreichende Internet-Anbindung zur Verfügung stand, die z. B. für Videokonferenzen und Kollaborationstools geeignet ist. Auch wenn ein Notebook im Haushalt dafür nutzbar war, konnte es bei mehreren schulpflichtigen Kindern schwierig sein, den Online-Schulunterricht zu organisieren. Vorteilhaft wäre es gewesen, wenn jedes Schulkind mit der (standardisierten) Hardund Software ausgestattet gewesen wäre, wie es seit Jahren in skandinavischen Schulen gehandhabt wird und nun auch in Deutschland zunehmend geschieht.

Ein weiteres Problem jedoch bestand und besteht in dem Angebot der Dienste, die häufig Mängel bezüglich des Datenschutzes und der Datensicherheit aufweisen. In der plötzlichen, durch Corona bedingten Schulschließsituation im März 2020 waren viele Schulen nicht handlungsfähig, selbst wenn landesweit über die Kultusministerien einige Hilfestellungen zu Auswahlkriterien und geeigneten Angeboten für das Homeschooling gegeben wurden. ${ }^{3}$ Zwar war man sich in vielen Fällen der abstrakten Kriterien bewusst, z. B. dass die Angebote datenschutzkonform sein sollten. Es war aber nicht jedem von Anfang an klar, dass dies auch bedeutete, sich mit Verschlüsselung beispielsweise bei Videokonferenzen, mit ServerStandorten innerhalb des Europäischen Wirtschaftsraums oder an anderen

3 Institut für Qualitätsentwicklung an Schulen Schleswig-Holstein (IQ.SH): Einsatz von digitalen Angeboten während der Corona-Krise. 2020. Online verfügbar unter: https://medienberatung.iqsh.de/corona2.html (Abfrage am: 21.09.2020). 
Orten mit angemessenem Datenschutzniveau, mit der Einbindung von Unterauftragnehmern, die keine zusätzlichen Risiken verursachen dürfen, und natürlich mit Geschäftsmodellen zu beschäftigen, die teilweise auf Analyse der Nutzerdaten (hier: Schülerinnen und Schüler) zielten. Einfacher schien es einigen Akteuren, die Kommunikation über (vermeintlich) bei allen Schülerinnen und Schülern verfügbaren Tools wie WhatsApp auf den Smartphones der Jugendlichen zu führen, was jedoch aus Datenschutzsicht alles andere als empfehlenswert war. Die Kritik der Datenschützer betrifft beispielsweise die nicht zu deaktivierende Synchronisation der (gehashten) Telefonnummern in den Adressbüchern der Smartphones mit den WhatsApp-Servern, sodass auch personenbezogene Daten von unbeteiligten Personen erfasst sind. Auch dass WhatsApp eine Tochter von Facebook und damit ein Datenaustausch unter beiden möglich ist, spricht gegen den Einsatz dieses Messengers.

Auf keinen Fall sollten Personen, die aus Datenschutzgründen kritische Angebote ablehnen, im schulischen Kontext dazu gezwungen werden hier dürfen keine Abstriche gemacht werden. Wenn verbreitete Lösungen, die aus Privatinteresse von einigen oder sogar vielen Schülerinnen und Schülern verwendet werden, nicht datenschutzkonform sind, dürfen sie nicht als Blaupause für den schulischen Einsatz dienen, zumal es vielfach bessere Angebote gibt oder man Veränderungen bei den Anbietern einfordern kann. In der Tat zeigte sich im Jahr 2020 während der Corona-Pandemie, dass auf Nachfrage mehr Datenschutz und Datensicherheit in Homeoffice- oder Homeschooling-Lösungen realisiert wurde. ${ }^{4}$

Standardisierte Lösungen für schulische E-Mail-Adressen für Lehrkräfte und für Schülerinnen und Schüler oder die Nutzung einer Schul-Cloud gab es zumindest nicht flächendeckend in Deutschland. Das Problem ist jedoch komplexer. Schnellschüsse oder ein unkritisches Implementieren jeder technischen Lösung sind nicht angeraten - aber man kann aus den

4 Jedoch sind auch Ende des Jahres 2020 Datenschutzanforderungen zum internationalen Datentransfer, wie sie der Europäische Gerichtshof $(\mathrm{EuGH})$ in seinem Urteil vom 16.07.2020, C-311/18, formuliert hat, bei Servern im außereuropäischen Ausland noch nicht überall umgesetzt. In dieser Entscheidung erklärte der EuGH das Abkommen „Privacy Shield“, das für viele Dienste als Rechtsgrundlage zum Austausch personenbezogener Daten mit den USA diente, für unwirksam und machte am Beispiel der Zugriffsmöglichkeiten für US-Nachrichtendienste deutlich, dass die Daten der europäischen Bürgerinnen und Bürgern nicht ohne bessere Garantien, z. B. einen ausreichenden Rechtsschutz, ins Ausland transferiert werden dürfen. 
folgenden Beispielen lernen, die heute schon in Deutschland oder anderswo Wirklichkeit sind oder es zumindest bald sein könnten.

\subsection{Dienstleister mit eigenen Interessen}

Im Bereich der Digitalisierung geht es nicht nur um das Schalten von Werbung, sondern um das Gewinnen von jahrelangen - vielleicht sogar lebenslangen - Kundinnen und Kunden: Schülerinnen und Schüler, die standardmäßig mit Hardware, Software und Nutzerkonten eines Anbieters ausgestattet werden, können durch Gewöhnung an das „Look \& Feel“ der User Interfaces auch nach ihrer Schulzeit die Dienste nutzen wollen. Das gilt umso mehr dann, wenn der Haushalt ebenfalls solche oder ähnliche Angebote nutzt, was vorteilhaft sein kann, wenn ansonsten die Geräte in der Schule verbleiben, aber man von zu Hause aus an seinen Projekten weiterarbeiten möchte. Außerdem kann es unbequem sein, wenn man den Anbieter wechseln möchte, nachdem man sich alles so konfiguriert hat, wie es einem am besten gefällt, und dort auch vielleicht weitere Daten gespeichert hat, die erst mühsam zusammengesammelt werden müssten. Deswegen läuft ein Kampf um die Kundenakquise im Klassenzimmer, bei dem große Anbieter sich auch über attraktive Angebote für Großaufträge oder andere Arten eines Sponsorings in Stellung bringen (siehe auch Harris 2016).

Wie auch in anderen Bereichen der Digitalisierung gewohnt, dominieren wenige globale Anbieter den Markt. Es fehlt in Deutschland und Europa an digitaler Souveränität. Problematisch ist eine wachsende Abhängigkeit von diesen wenigen Anbietern. Für öffentliche Stellen hat insbesondere Microsoft mit seiner Office-Umgebung eine Quasi-Monopol-Funktion, sodass es Alternativen schwer haben - auch in der Schule (Kuketz 2020). Apple wiederum stellt „Apple Education Specialists“ zur Verfügung, die umfassend beraten, wie Digitalisierung im Schulbereich funktioniert - natürlich mit Angeboten von Apple. ${ }^{5}$

Auch wenn es nicht um die Grundausstattung der Schülerinnen und Schüler geht, kommt man für die Anwendungen und Online-Angebote in eine ähnliche Situation, denn Anbieter für Videos (wie YouTube) oder für Suchmaschinen (wie Google oder Bing) verfolgen mit den Nutzungsdaten eigene Zwecke. Auch sind viele Webseiten mit Tracking-Tools ausgestattet,

5 https:/www.apple.com/de/education/how-to-buy/education-specialist/ (Abfrage am: 21.09.2020). 
die wiederum bestimmte Daten der Schülerinnen und Schüler erfassen und auswerten können. Zudem sind die Kinder und Jugendlichen der Internet-Werbung ausgesetzt, wenn dies nicht aktiv - z. B. durch den Einsatz von Werbeblockern - unterbunden wird.

\section{2 Überwachungsaufrüstung}

Überwachungsfreie Räume werden immer seltener - das gilt auch für Schulen. Während in Deutschland vielfach genau abgewogen wird, unter welchen Bedingungen in welchen Zeiten welche Bereiche des öffentlichen Raums von einer Videoüberwachung umfasst sein dürfen und im Schulbereich auch Schulträger, Lehrkräfte, Eltern oder die Schülervertretung mitdiskutieren, wird dies in anderen Ländern häufig nicht infrage gestellt. Mittlerweile beschäftigen sich einige Untersuchungen mit dem Effekt, den eine Überwachung auf Schülerinnen und Schüler oder auch auf Studierende haben (Lindstrom et al. 2018, Birnhack/Perry-Hazan 2020): teilweise werden Videoüberwachungen aus Sicherheitssicht begrüßt, aber aus Datenschutzgesichtspunkten sehen viele Personen darin einen Eingriff in ihre Privatsphäre.

Fälle von heimlicher Überwachung, beispielsweise über ausgegebene Schul-Notebooks per Webcam ins Kinderzimmer, werden nicht nur in Deutschland besonders kritisch gesehen (Zips 2010). Ähnliches gilt für Situationen, in denen Kinder und Jugendliche andere Schülerinnen und Schüler oder Lehrkräfte heimlich per Smartphone aufnehmen und durch Verbreiten der Aufnahmen lächerlich machen.

Jedoch geht die Überwachung gar nicht in allen Fällen von den Schulen oder Schülerinnen und Schülern aus. So statten einige Eltern ihre Grundschulkinder mit Smartwatches aus, die mit einer Funktion zum Fernaktivieren der Tonübertragung („Remote Voice Monitoring“) ausgestattet sind. Damit wollen sie bei Bedarf in den Unterricht hineinlauschen und überprüfen, ob ihre Sprösslinge fair von Lehrkräften oder Mitschülerinnen und Mitschülern behandelt werden. Manchmal mischen sich die Eltern aus der Ferne auch direkt ein: Die Smartwatch überträgt dann ihre Stimme in den Klassenraum. An vielen Schulen hat man den Einsatz solcher Geräte untersagt; ${ }^{6}$ falls Kinder doch damit ausgestattet sind, müssen sie die

6 Zum Beispiel: Niedersächsische Landesschulbehörde: Smartwatches im Schulalltag: Eine (datenschutzrechtliche) Herausforderung. Online verfügbar unter: https://www.la ndesschulbehoerde-niedersachsen.de/themen/schulorganisation/datenschutz/daten 
Smartwatches für die Unterrichtszeit abgeben. Generell unterfallen Geräte mit heimlicher Abhörfunktion der Kategorie der verbotenen Sendeanlagen. ${ }^{7}$

Mit dem Homeschooling stellen sich Fragen, ob auch in die Zimmer der Kinder und Jugendlichen hineingeschaut werden darf, um festzustellen, ob weitere Personen anwesend sind und unbefugte Hilfestellung geben könnten. Zumindest für das Ablegen von Prüfungen wurde dies bereits an Hochschulen praktiziert, jedoch darf man bezweifeln, dass dies auf Basis einer gültigen Einwilligung geschehen ist (Schneider 2020).

Eine Überwachung kann technisch auch über besondere Tracking-Apps erfolgen, die die Lernenden auf ihrem Smartphone installieren. Im Fall einer US-amerikanische Hochschule sollte anhand der App „SpotterEDU“ festgestellt werden, inwieweit die Studierenden den Unterricht schwänzten, zu spät kamen oder zu früh gingen. Außerdem ließ sich feststellen, inwieweit die Studierenden miteinander interagierten oder bestimmte Orte mieden. Man wollte Einzelgänger herausfinden, die vielleicht psychische Probleme hatten, oder aus dem Umstand, dass jemand nie die Cafeteria aufsuchte, ableiten, dass eine Essstörung vorliegen könne (Harwell 2019).

Noch Zukunftsmusik für deutsche Schulen sind Gehirnanalysen per EEG, die angeblich ein individuell zugeschnittenes Lernen ermöglichen sollen. Bedenken bestehen aber, wenn man die Aufmerksamkeit der Schülerinnen und Schüler immer mehr kontrolliert, um Tagträumereien und abschweifende Gedanken einzufangen und die Lernleistung zu optimieren (Tangens 2020). Selbst wenn ein "Gedankenlesen" durch Technik weit weg erscheint, wird an einer Analyse von Reaktionen und Emotionen der Personen gearbeitet. Schon in der Praxis eingesetzt - wenn auch noch nicht in deutschen Schulen - werden Analysesysteme, die Gesichtsausdrücke, Augenbewegungen oder die Stimme auswerten können (Hansen 2021). Dies kann zur Unterstützung der Menschen dienen, birgt aber auch das Risiko einer Manipulation, wenn aus den Daten hervorgeht, wie man z. B. eine positive Reaktion einer Person hervorrufen kann oder worauf sie schnell gestresst reagiert.

schutz-im-schulalltag/smartwatches-im-schulalltag-eine-datenschutzrechtliche-hera usforderung-1 (Abfrage am: 21.09.2020).

7 Bundesnetzagentur: Missbrauch von Sendeanlagen - Hinweise zu einzelnen Produktkategorien. Online verfügbar unter: https:/www.bundesnetzagentur.de/DE/Sachgebie te/Telekommunikation/Unternehmen_Institutionen/Anbieterpflichten/Datenschu tz/MissbrauchSendeanlagen/HinweiseProduktkategorien/hinweiseproduktkategori en-node.html (Abfrage am: 21.09.2020). 


\section{3 (Un-)Faire Entscheidungen durch Künstliche Intelligenz}

Im Schulbereich wird angestrebt, schul- und regionenübergreifend vergleichbare Bewertungen der Leistungen zu gewährleisten. Hier kommen mittlerweile algorithmische Systeme, teilweise basierend auf Methoden der Künstlichen Intelligenz, zum Einsatz. In Großbritannien zeigte sich damit jedoch ein Problem, denn in fast $40 \%$ der Fälle wurden die Leistungen der Schülerinnen und Schüler heruntergestuft (Quinns/Adams 2020). Zwar hätte der Einsatz von Algorithmen sogar das Potenzial zu mehr Gerechtigkeit, als dies bei Bewertungen durch menschliche und vorurteilsbehaftete Lehrkräfte der Fall ist. Jedoch zeigt sich immer wieder, dass der Einsatz von Künstlicher Intelligenz inhärente Verzerrungen aus der realen Welt nicht ausgleicht, sondern vielmehr verstärkt. Wenn beispielsweise in dem Vorjahr der schlechteste Schüler von Schule X kommt, darf daraus nicht - wie es wohl im britischen Bewertungssystem der Fall war - folgen, dass im aktuellen Jahr wieder ein Schüler an der Schule X die schlechteste Note erhält. Gute Schülerinnen und Schüler, die öffentliche Schulen in ärmeren Bezirken Londons besuchten, hatten bei diesem Algorithmus gar keine Chance, leistungsgerecht bewertet zu werden.

Trotz dieses Debakels wird der künstlichen Intelligenz eine große Rolle in der Schule der Zukunft zugeschrieben. Dies betrifft nicht nur (möglichst faire) Bewertungen, sondern auch das differenzierte und individualisierte Lernen, bei dem das technische System passgenau auf die Fähigkeiten und Potenziale der Lernenden eingehen kann. Im Vergleich dazu fehlt es Lehrkräften an der Zeit oder manchmal auch an der nötigen Geduld für genau auf die jeweilige Person zugeschnittene Wissensvermittlung. Dies betrifft auch Menschen mit Behinderungen, die von besonderer Unterstützung profitieren. Hier stellen sich jedoch nicht nur Datenschutzfragen: Beispielsweise kann der Mehrwert einer vertrauensvollen Lehrer-SchülerBeziehung (siehe Abschnitt 3.3), die auch von der sozialen Interaktion lebt, damit nicht ersetzt werden.

\section{Ergebnisse und Schlussfolgerungen}

Dass Fortschritte bei der Digitalisierung in den Schulen notwendig sind, wird keiner bezweifeln. Natürlich ist dafür eine Grundausstattung an Hardware, Software und ausreichender Internet-Anbindung sowohl in den Schulen als auch zu Hause bei den Lehrkräften sowie Schülerinnen und Schülern erforderlich. Empfehlenswert ist eine einheitliche schulische Ausstattung, da ansonsten einige Personen Vor- und andere Nachteile in der 
Nutzung haben könnten. Mindestens genauso wichtig ist es, die Lehrkräfte für die Digitalisierung und ihre Chancen und Risiken zu sensibilisieren und auszubilden.

Einige Basisdienste - beispielsweise schulische E-Mail-Adressen, digitale Kalender oder Dokumentablagen - sollten generell für alle bereitgestellt werden: Hier sind die Kultusministerien gefragt, und in mehreren Bundesländern läuft dies auch schon gut. Man muss hier zusätzlich berücksichtigen, dass es nicht nur eine Frage der Technikentwicklung ist, sondern auch der Betrieb geregelt ablaufen muss, z. B. wenn die Nutzerinnen und Nutzer ihre Passwörter vergessen, dass man ein Verfahren für neue und ausgeschiedene Nutzende benötigt und wie man auf gemeldete Datenpannen reagiert. Auch stellt sich die Frage nach der betreibenden Instanz: ein öffentliches Rechenzentrum, das Kultusministerium oder ein privater Anbieter. Man muss sich auch überlegen, ob nur Lehrkräfte und Schülerinnen und Schüler oder auch Sozialarbeiterinnen und Sozialarbeiter in den Schulen oder Eltern ebenfalls darüber angebunden sein sollen.

Auch wenn der Schulbereich Ländersache ist, steht dies einer länderübergreifenden Kooperation beim Konzipieren und Implementieren von Lösungen nicht entgegen. Auch innerhalb der Bundesländer können sich die Schulen mit ihren Erfahrungen austauschen und von Synergien profitieren.

Bei der Auswahl von Dienstleistern und Anbietern darf nicht aus dem Blick geraten, dass Schulen aus wirtschaftlicher Sicht - Kaufkraft, Kundenbindung - interessant sind, aber man genau deswegen jede Abhängigkeit oder gar Beeinflussung der Schülerinnen und Schüler vermeiden sollte. Eigene Entwicklungen, z. B. auf Open-Source-Software, können einen Beitrag zur digitalen Souveränität leisten, die der Bund und die Länder ebenso wie Europa anstreben.

Für die Schulen für Ort gilt es, sich klare Regeln zu geben (oder auch von den Kultusministerien einzufordern), welche Tools und Anwendungen unter welchen Bedingungen eingesetzt werden können, besonders im Bereich der Schulverwaltung und für schulische Veranstaltungen. Sollen beispielsweise eigene Endgeräte (Bring-your-own-device) genutzt werden, und wie werden die Geräte gegen unberechtigte Zugriffe geschützt? Wer kümmert sich um Updates oder um die Wartung von Hard- und Software? Welche Kommunikationsmittel sollen auf Klassenfahrten Verwendung finden, z. B. mit Mobiltelefonen? Dürfen Lehrkräfte bestimmte Kommunikation ausschließen, z. B. wenn sie von Eltern über private Adressen, etwa per WhatsApp, kontaktiert werden? Wie kann und soll ein Fernunterricht technisch unterstützt werden? Sollen spezielle digitale Hilfsmittel für individualisiertes Lernen zum Einsatz kommen, die mehr Daten über die 
Schülerinnen und Schüler auswerten? Wann benötigt man eine Einwilligung der Eltern oder der Jugendlichen? Dies alles ist auch wichtig, um den Lehrkräften Sicherheit für ihr Tun zu geben und sie vor überbordenden Erwartungen beispielsweise der Eltern zu schützen.

Für den pädagogischen Bereich - im Unterricht - ist ein breiterer Blick auf die verfügbaren Angebote wesentlich, beispielsweise indem man nicht nur eine Suchmaschine oder eine Quelle in den Vordergrund stellt, sondern kritisch den Einsatz verschiedener Angebote beleuchtet. Dazu gehört auch, dass man sich im Unterricht mit Datenschutzerklärungen oder Allgemeinen Geschäftsbedingungen beschäftigt, über Klarnamenpflicht, Pseudonyme und anonyme Nutzung diskutiert und praktische Erfahrungen im Selbstdatenschutz macht. Es bestehen zahlreiche Angebote von Medienanstalten, Polizei, Verbraucherschutz und Datenschützern, die bei Bedarf von Schulen angefordert werden und spezifisch für Klassen, Schulentwicklungstage der Lehrkräfte, Projektwochen oder Elternabende zugeschnitten sein können. ${ }^{8}$

8 Beispielsweise die Angebote des Medienscouts e. V., http://medienscout.info/, des Verbraucherzentrale Bundesverband (vzbv) e. V., https://www.verbraucherbildung. de/suche/materialkompass, der Initiative „Datenschutz geht zur Schule“ des Berufsverbands der Datenschutzbeauftragten Deutschlands (BvD) e. V., https://www.bvd net.de/datenschutz-geht-zur-schule/, oder auch Veranstaltungen in den einzelnen Bundesländern wie der Datenschutzakademie Schleswig-Holstein, https://www.dat enschutzzentrum.de/akademie/, oder des Offenen Kanals Schleswig-Holstein, https://www.oksh.de/mitmachen/lernen/medienkompetenz-mk-fuer-schule/ (Abfrage am: 21.09.2020). 


\section{Literatur}

Birnhack, Michael / Perry-Hazan, Lotem (2020): School Surveillance in Context: High School Students' Perspectives on CCTV, Privacy, and Security. In: Youth \& Society 52 (7), S. 1312-1330.

Bitkom (2020): Corona-Note „mangelhaft": Eltern gehen mit Schulen hart ins Gericht. Pressemitteilung des Bitkom vom 14.09.2020. Online verfügbar unter: https://w ww.bitkom.org/Presse/Presseinformation/Corona-Note-mangelhaft-Eltern-gehe n-mit-Schulen-hart-ins-Gericht (Abfrage am: 21.09.2020).

Bitkom Research (2020): Digitale Schule in Corona-Zeiten. Präsentation von Achim Berg, Bitkom-Präsident. Online verfügbar unter: https://www.bitkom.org/sites/d efault/files/2020-09/prasentation-bitkom-pk-digitale-schule-in-corona-zeiten-14-0 9-2020_final.pdf (Abfrage am: 21.09.2020).

Förtsch, Matthias (2018): Diktatur des Datenschutzes. Kolumne. Das Deutsche Schulportal. 23.04.2018. Online verfügbar unter: https://deutsches-schulportal.de/kolu mnen/zukunft-der-schule-diktatur-des-datenschutzes/ (Abfrage am: 21.09.2020).

Fraillon, Julian / Ainley, John / Schulz, Wolfram / Friedman, Tim / Duckworth, Daniel (2020): Preparing for Life in a Digital World - IEA International Computer and Information Literacy Study 2018 International Report. International Association for the Evaluation of Educational Achievement (IEA). Springer.

Hansen, Marit (2021): Private Haushalte. In: Hornung, Gerrit / Schallbruch, Martin (Hg.): IT-Sicherheitsrecht. Praxishandbuch. Nomos, S. 620 ff. Im Erscheinen.

Harris, Ainsley (2016): How Google Is Schooling Apple And Microsoft In the Battle For America's Classrooms. Fast Company, 12.09.2016. Online verfügbar unter: https:// www.fastcompany.com/3062958/how-google-is-schooling-apple-and-microsoft-i n-the-battle-for-americas-classrooms (Abfrage am: 21.09.2020).

Harwell, Drew (2019): Colleges are turning students' phones into surveillance machines, tracking the locations of hundreds of thousands. In: The Washington Post, 24.12.2019. Online verfügbar unter: https://www.washingtonpost.com/technolo gy/2019/12/24/colleges-are-turning-students-phones-into-surveillance-machines- $t$ racking-locations-hundreds-thousands/ (Abfrage am: 21.09.2020).

Kuketz, Mike (2020): Bildungswesen: Entlarvung der häufigsten Microsoft-Mythen. Blog-Beitrag. Online verfügbar unter: https://www.kuketz-blog.de/bildungswese n-entlarvung-der-haeufigsten-microsoft-mythen/ (Abfrage am: 21.09.2020).

Lautebach, Urs (2018): Informatik für alle! Ein Plädoyer. 01.02.2020. Online verfügbar unter: https://gi.de/themen/beitrag/informatik-fuer-alle-ein-plaedoyer-1 (Abfrage am: 21.09.2020).

Lindstrom Johnson, Sarah / Bottiani, Jessika / Waasdorp, Tracy E. / Bradshaw, Catherine P. (2018): Surveillance or Safekeeping? How School Security Officer and Camera Presence Influence Students' Perceptions of Safety, Equity, and Support. In: Journal of Adolescent Health 63 (6), S. 732-738. 
Quinn, Ben / Adams, Richard (2020): England exams row timeline: was Ofqual warned of algorithm bias? In: The Guardian, 20.08.2020. Online verfügbar unter: https:/www.theguardian.com/education/2020/aug/20/england-exams-row-timeli ne-was-ofqual-warned-of-algorithm-bias (Abfrage am: 21.09.2020).

Schneider, Marcel (2020): Jura-Klausuren aus dem Homeoffice. In: Legal Tribune Online, 19.03.2020. Online verfügbar unter: https:/www.lto.de/recht/studium-refer endariat/s/corona-virus-jurastudium-klausuren-homeoffice-bucerius-law-school-d ebatte-taeuschung/ (Abfrage am: 21.09.2020).

Tangens, Rena (2020): Big Brother Awards 2020: Preisträger Firma Brainco und der Leibniz-Wissenschaftscampus der Uni Tübingen. Laudatio am 18.09.2020. Online verfügbar unter: https://bigbrotherawards.de/2020/bildung-brainco-und-leibnizwissenschaftscampus-tuebingen (Abfrage am: 21.09.2020).

Verbraucherzentrale Bundesverband (vzbv) e. V. (2020): Keine Werbung in der Schule. Aktivitäten von Wirtschaft im Bildungsbereich wirksam begrenzen und kontrollieren. Positionspapier der Verbraucherzentralen und des Verbraucherzentrale Bundesverbands e. V., 05.03.2020. Online verfügbar unter: https://www.vzbv.de/ sites/default/files/downloads/2020/03/11/20-03-05_wirtschaft_in_schule_ak_vz_p ositionspapier_und_anhang.pdf (Abfrage am: 21.09.2020).

Zips, Martin (2010): USA: Kamera in Schul-Laptops. Per Webcam direkt ins Kinderzimmer. In: Süddeutsche Zeitung, 23.02.2010. Online verfügbar unter: https://www. sueddeutsche.de/karriere/usa-kamera-in-schul-laptops-per-webcam-direkt-ins-kin derzimmer-1.20027 (Abfrage am: 21.09.2020). 
